\title{
THE COSMIC X-RAY BACKGROUND
}

\author{
R. J. WEYMANN \\ (Steward Observatory, University of Arizona, Tucson, Ariz., U.S.A.)
}

Evidence for a diffuse, isotropic non-terrestrial component of X-ray radiation has been accumulating since the very beginnings of $\mathrm{X}$-ray astronomy. This evidence now seems secure beyond reasonable doubt, especially in the light of recent experiments, e.g. one in which a very clean separation from the cosmic ray and terrestrial background has been achieved (Pounds, 1967), and another in which a counting rate proportional to the solid angle of the sky subtended to three different detectors was found (Matsuoka et al., 1967).

In this very brief review I wish simply to discuss the current ideas on the mechanisms for the production of the background and the extent to which the present observations can discriminate between them and to consider the information obtainable from the very soft X-ray region, which so far has been relatively little exploited.

The status of the observations has been summarized in Fig. 1 of a recent excellent review by Gould (1967). More recent observations do not change the over-all picture except that the tendency for the lower energy range (1-20 keV) to have a slightly flatter number-spectral index $(\sim 1 \cdot 7)$ than the higher energy range $(20 \mathrm{keV}-1 \mathrm{meV}$ : $\sim 2 \cdot 2$ ) seems strengthened. All groups agree that except for a group of partially unresolved sources in the direction of the galactic centre, any anisotropy present must amount to less than about $10 \%$. In particular, in the plane of the galaxy no firm evidence for a general brightening has been found.

As is almost self-evident from Gould's Fig. 1, thermal bremsstrahlung cannot explain the whole range of the background, and accordingly attention has centered on non-thermal processes - in particular Compton scattering of photons by highenergy electrons. The high degree of isotropy of the background, together with the demonstration by Felten and Morrison (1966) that galactic electrons cannot account for the background, suggest that an extragalactic origin be sought, and these have divided into two classes:

(a) A smooth continuous background arising from universal extragalactic highenergy electrons interacting with the $3^{\circ} \mathrm{K}$ photons.

(b) Many discrete extragalactic sources.

One could try to differentiate between these two possibilities along any of the following three lines:

(1) the shape of the observed spectrum,

(2) the total-power requirements,

Perek (ed.), Highlights of Astronomy, 220-227. (c) I.A.U. 
(3) the degree of isotropy of the radiation.

The continuum models proposed can be subdivided according to the mode of providing the high-energy electrons: Felton and Morrison suggest direct replenishment by active radio sources, whereas Gould has suggested secondary production via interaction of a postulated intergalactic cosmic-ray component with intergalactic matter, the origin of the high-energy protons being unspecified. In either case, one requires a production spectrum for the electrons which, following the steepening due to Compton losses, gives rise to the observed X-ray spectrum. On the basis of the clustering of radio spectral indices about the value $0 \cdot 7$, one is led to an index of 2.2 in the (number) spectral index for the X-rays; estimates for the secondary production mechanism based upon the observed cosmic-ray spectrum lead to about the same value for the spectral index, which lies nicely in the observed range.

This is taken to be a strong point in favor of the continuum models; on the other hand, some sources in our own galaxy, notably the Crab, have indices of about $2 \cdot 2$ in the $1-100 \mathrm{keV}$ range - also just in the range of the observed spectral index. No data on the spectral index of M 87 in the X-ray region itself are available as yet, but the combined radio, optical, and X-ray data are consistent with an index of 1.7 again not incompatible with the data over the low-energy $\mathrm{X}$-ray range.

At the present time, therefore, we feel that there is no basis for discriminating between the two models on the basis of spectra.

Regarding the total power requirements, and neglecting for a moment evolutionary considerations, both the discrete and continuum models run into some difficulties. The input of relativistic particles into intergalactic space was estimated by Felten and Morrison by supposing that the entire store of these particles (whose total energy store is estimated by assuming equipartition of the field and particle energies) is turned over to intergalactic space every $10^{6}$ years. Taking a typical store of highenergy electrons to be $10^{57}$ ergs and using a density of strong sources not very much different from that recently given by Schmidt (1966), one finds that, over $10^{10}$ years, the store of particles fails by about 2 orders of magnitude to explain the X-ray flux; as remarked by these authors, the assumption of a field lower than the equipartition value increases the energy of relativistic particles and hence the input. But the other side of the coin is that the total energy requirements, already very large $-10^{59}$ ergs in relativistic particles if 100 times the electron energy in heavy particles is assumed, turned over $10^{3}$ times during the lifetime of $10^{9}$ years estimated by Schmidt (1966), amounts to $10^{62} \mathrm{ergs}-$ go up even further.

The situation is equally uncomfortable for the discrete source models. We consider three types of objects radiating in the 1-10 $\AA$ band at three different levels:

(1) Objects with a flux $10^{46} \mathrm{ergs} / \mathrm{sec}$ - the flux inferred for $3 \mathrm{C} 273$ if at its cosmological distance (Friedman, 1967),

(2) Objects with a flux of $2 \times 10^{43} \mathrm{ergs} / \mathrm{sec}$ - based upon M87 (Friedman, 1967),

(3) Objects with a flux of $2 \times 10^{39} \mathrm{ergs} / \mathrm{sec}$ which might be appropriate for objects 
like our own galaxy - equivalently we might suppose that each gram of ordinary condensed matter radiates at a rate of $10^{-5} \mathrm{erg} / \mathrm{g}$.

The specific intensity in the $1-10 \AA$ band seems to lie between about $3 \times 10^{-8}$ and $1.0 \times 10^{-7} \mathrm{ergs} / \mathrm{cm}^{2} / \mathrm{sec} / \mathrm{ster}$, and this is related to the source density and luminosity per source by the relation

$$
I \sim k \frac{c}{H_{0}} n_{0} \frac{L_{v}}{4 \pi},
$$

where $H_{0}$ is the Hubble constant, $n_{0}$ the density of sources, and $L_{v}$ the luminosity of the source.

The constant $k$ depends upon the spectral index and the cosmological model; for $q_{0}=\frac{1}{2}$ and a photon spectral index of 2.5 it is $\frac{1}{3}$; for an index of 2.0 it is $\frac{2}{5}$. Table 1 summarizes the density of various classes of objects required to produce the observed flux, together with the estimated actual densities. For purposes of a discussion below we also give the required number of objects per square degree out to a redshift of $0 \cdot 6$.

\section{Table 1}

Class of objects

Ordinary

condensed matter

Strong radio

sources like M 87

Quasars
Required density

$$
\begin{aligned}
& 1.3 \times 10^{-29} \text { to } 4 \times 10^{-29} \mathrm{gm} / \mathrm{cm}^{3} \\
& 1.9 \times 10^{-4} \text { to } 5.8 \times 10^{-4} \mathrm{obj} . /(\mathrm{mpc})^{3}
\end{aligned}
$$$$
4 \times 10^{-7} \text { to } 1.2 \times 10^{-6} \mathrm{obj} . /(\mathrm{mpc})^{3}
$$

Estimated density

$7 \times 10^{-31} \mathrm{gm} / \mathrm{cm}^{3}$

$7 \times 10^{-6} \mathrm{obj} . /(\mathrm{mpc})^{3}$

$2 \times 10^{-9} \mathrm{obj} . /(\mathrm{mpc})^{3}$
Required no./ deg. ${ }^{2}$ to $Z=0.6$

$1.1 \times 10^{2}$

$2.3 \times 10^{-1}$

a Based upon each object having $M=10^{11} M_{\odot}$.

Evidently all three major classes fail by about $1 \cdot 5-2 \cdot 0$ orders of magnitude to account for the flux - about the same margin as the Felten-Morrison hypothesis. Other sources could of course be added to the list - e.g. Sandage (1967) estimates that the number of 'radio-quiet quasars' are 500 times more numerous than the quasars themselves - but the very fact that they are radio-quiet suggests a deficiency of high-energy electrons and hence, perhaps, of X-rays.

Before considering possible modifications to the foregoing due to evolutionary effects, we consider a third possible discriminant: suppose that the background does in fact consist of discrete sources - under what circumstances might we expect to 'resolve' the sources? We consider some numerical examples based upon the following model:

(a) The sources are all identical and are randomly distributed throughout the universe, assumed to be characterized by $q_{0}=\frac{1}{2}$,

(b) The (energy) spectral index is 1.5 ,

(c) Each source radiates at a constant rate, but there are no sources beyond $z=15$. 
How might one distinguish between two models in which nearly all the flux comes from a continuum (but with a few nearby resolved sources) from one in which it all comes from discrete point sources? Figure 1 shows the cumulative probability distributions for flux levels from random directions in the sky, normalized to the median flux level. Due to instrumental errors and photon statistics, even a smooth continuum would be 'broadened' from a step function - this is represented by the integral of a gaussian distribution with a probable error of $10 \%$ and labeled ' $\mathrm{GS}$ '. The other curves are the distributions in flux (uncorrected for instrumental effects) for cases in which the density of sources is such that 2,16 , and 128 sources out to a redshift of 0.6 are contained in the beam. This example suggests that for $N$ much larger than 128 $(N \geqslant 500)$ it would be very difficult to distinguish between the 'discrete' and 'nearly

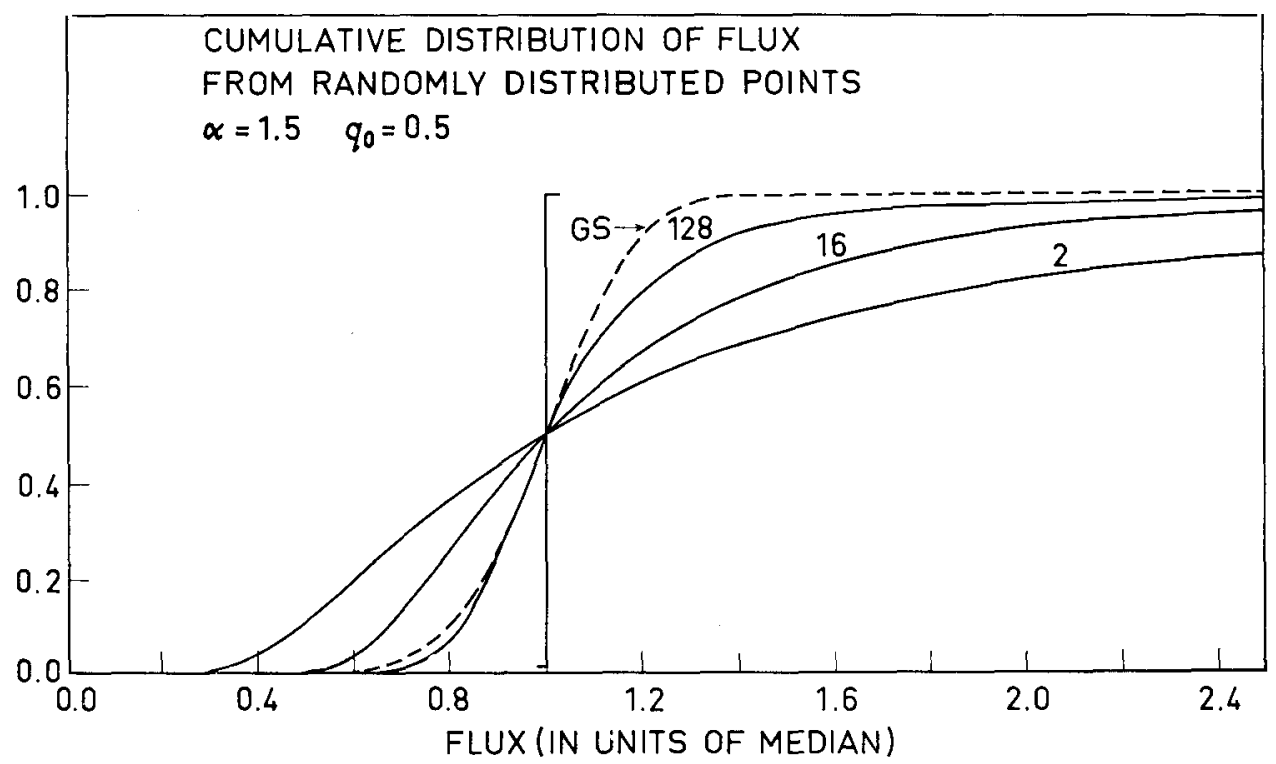

FIG. 1. The probability that a random sample of sky will yield a flux less than a certain value, in units of the median value of the flux. Point sources radiating with a spectral index of 1.5 in a flat cosmological model were considered. The curves labeled 128,16, and 2 correspond to the mean number of sources out to $z=0.6$ in the beam. The curve 'GS' is a Gaussian distribution with a probable error of $10 \%$.

continuous' models on the basis of resolvability. Realizing that background experiments have been carried out with solid angles ranging from $10^{1}$ to $10^{3}$ square degrees, one sees from Table 1 that even a class of M 87-type objects could not be resolved if they existed in numbers sufficient to explain the background, although the quasar-like objects could be.

Let us now consider what sort of evolutionary considerations are required to avoid the difficulties with both the discrete and continuum models. 
This question has recently been discussed by, among others, Bergamini et al. (1967). Their work may be summarized as follows:

(a) Rather than make semi-empirical estimates of the X-ray flux from various classes of objects as we have done above, they proceed directly from the ratio of synchrotron to Compton radiation arising from the $3^{\circ}$ radiation, which is known if the magnetic field is specified.

(b) Specific account is taken of the increase in temperature and energy density of the $3^{\circ} \mathrm{K}$ radiation field - this has the consequence that the ratio of Compton radiation to synchrotron radiation increases sharply with increasing red-shift. On the other hand, other things being equal, the severe Compton losses will cause the break in the electron-energy spectrum, and hence in the Compton and synchrotron spectrum, to occur at rather low energies for these distant sources.

(c) The interpretation of the radio-source counts seems to demand a rather strong increase in the activity of a class of strong sources with increasing red-shift (Longair, 1966; Schmidt, 1967), and this will further enhance the contribution of distant sources.

(d) The specific model used for the radio sources is one in which electrons are continuously injected with a power-law spectrum but then escape after a fixed time $T_{0}$. The magnetic field in the sources is supposed constant in time and must be a few microgauss. The confinement time is chosen in such a way that the break in the synchrotron spectrum occurs outside the observed region, where no break is observed. If it were to occur for very low frequencies, uncomfortably long confinement times would be required.

The size of these evolutionary effects can be seen by considering sources with $H=10^{-6}$ gauss, $T_{0}=3 \times 10^{6}$ years, injection spectral index for the electrons of 2.4 , and a flat $\left(q_{0}=\frac{1}{2}\right)$ cosmological model with no sources beyond $Z_{\max }=4$. Consider three situations: (a) No account is taken of either the increase in the $3^{\circ}$ radiation field, or increased activity of the sources, (b) no increase in the activity of the sources is considered, but the effects of the increased background radiation, both in enhancing the Compton radiation and in affecting the electron-energy spectrum, are considered, (c) the probability of sources occurring in a given sample of objects increases as $(1+Z)^{5 \cdot 7} ; 5 \cdot 7$ and the cut off $Z_{\max }=4.0$ are chosen simply because these parameters were found by Longair to be consistent with the radio-source counts.

At about $1 \mathrm{keV}$, model (b) yields an intensity about 15 times that of model (a), while model (c) in turn yields about 1500 times the intensity of model (b). Finally, if we arbitrarily divide all sources into two classes - those which evolve as $(1+Z)^{5 \cdot 7}$ and those which do not - then this model accounts for the observed background flux if only $2 \%$ of the radio emission per unit volume at the present epoch comes from the strongly evolving class. Longair tentatively identified his strongly evolving sources with the QSS, and both the steep index of 5.7 and the figure of $2 \%$ are not inconsistent with recent results of Schmidt (1967). This model predicts a spectral index of $1 \cdot 7$ in the soft $\mathrm{X}$-ray region and $2 \cdot 2$ in the hard region with a broad transition region centered about $3 \mathrm{keV}$. 
If the QSS are indeed cosmological, it seems possible therefore that the X-ray background may be produced by these objects or their immediate successors, although the model used above is certainly much too simple. It will be interesting to repeat the resolvability calculation described above for this model, for the larger contribution by the numerous distant objects will make resolution more difficult. Moreover,

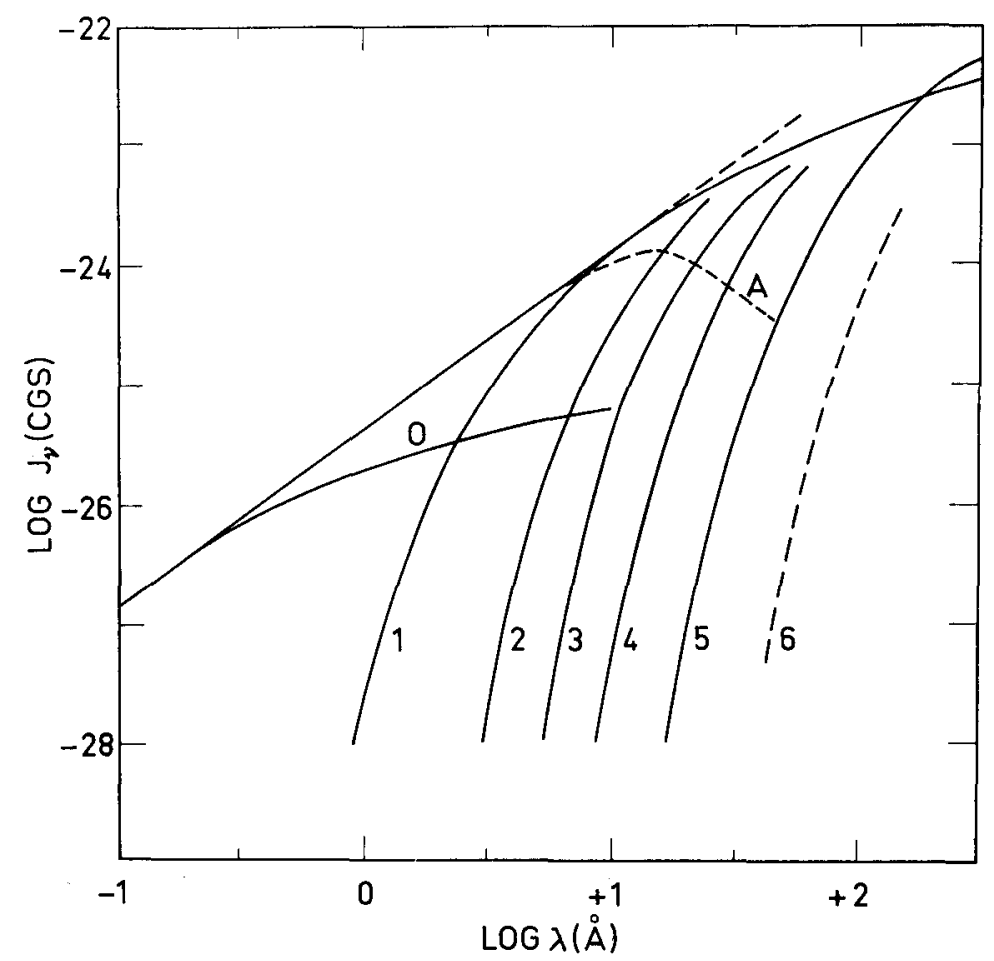

FIG. 2. Emission and absorption by intergalactic gas in the soft $X$-ray region. Curves $1-5$ show the emission expected from a hot gas with present density $10^{-5}$ particles $/ \mathrm{cm}^{3}$ with five different thermal histories all satisfying the Gunn-Peterson criterion on the absence of $L y$ - $\alpha$ absorption at $z \sim 2$. Curve 6 does not satisfy this criterion. Curve ' $A$ ' is the background resulting from a uniform distribution of neutral hydrogen in which is embedded a uniform distribution of $X$-ray emission with a spectral index of 1.5 . Curve ' $O$ ' is an approximate representation of the observations.

the electrons will continue to Compton scatter after being disgorged from the sources as in the Felten-Morrison model, and a quasi-continuum background will arise which may make a significant contribution to the total background.

Let us now turn to information which might be obtainable from observations of the X-ray background in the soft X-ray region - from, say, 1.0 to $0.25 \mathrm{keV}$.

In the first place, it will certainly be of interest to see whether the gradual leveling off of the flux, suggested by the most recent experiments, holds up. But there are also 
interesting possibilities for obtaining information about intergalactic matter in either neutral or ionized form. Aside from its intrinsic interest, the question of intergalactic matter touches directly upon the mechanism for the background proposed by Gould which requires on the order of $10^{-5}$ particles $/ \mathrm{cm}^{3}$.

Detection of thermal bremsstrahlung from a hot, ionized gas with densities around $10^{-5}$ particles $/ \mathrm{cm}^{3}$ might be achieved. Curves 1-5 of Figure 2 show the emission from an intergalactic gas with several plausible thermal histories, all satisfying the Gunn-Peterson restriction on $\mathrm{Ly}-\alpha$ at $Z \sim 2 \cdot 0$. Curve 6 is the emission from a case which does not satisfy this restriction. Evidently the critical observational requirement is to push the observations to very low energies, well into the region where galactic absorption is expected to become important. The possibility of detecting neutral hydrogen by X-ray absorption has been frequently discussed. Although not nearly as sensitive as the $\mathrm{Ly}-\alpha$ absorption in the QSO, the Ly- $\alpha$ test is still plagued by the uncertainty in the local vs. cosmological issue. Curve ' $A$ ' in Figure 2 shows the resulting background for a uniform distribution of sources radiating with an energy spectral index of 1.5 (straight line with dashed extension), intermixed with a uniform distribution of neutral hydrogen. Note the decrease is not exponential, but would tend to become so if the emission comes mostly from distant sources as described above. For pure hydrogen, optical-depth unity occurs for a photon whose final wavelength is $10 \AA$; for a normal admixture of helium this is shifted to $6 \AA$. This model evidently cannot explain the apparent flattening of the background, indicated by ' $O$ ', but obviously much more work with moderate spectral resolution needs to be done in the soft $\mathrm{X}$-ray region before any conclusions about intergalactic matter can be drawn.

\section{References}

Bergamini, R., Londrillo, P., Setti, G. (1967) preprint.

Felten, J.E., Morrison, P. (1966) Astrophys. J., 146, 686.

Friedman, N. (1967) Paper read at Joint Discussion on X-Ray Astronomy, 1.A.U., Prague.

Gould, R.J. (1967) Amer.J. Phys., 35, 376.

Longair, M.S. (1966) Mon. Not. Roy. astr. Soc., 133, 421.

Matsuoka, M., Oda, M., Ogawara, Y., Hayakawa, S., Kato, T. (1967) preprint.

Pounds, K. (1967) Paper read at Joint Discussion on X-Ray Astronomy, I.A.U., Prague.

Sandage, A.R. (1967) Invited discourse, I.A.U., Prague.

Schmidt, M. (1966) Astrophys. J., 146, 7.

Schmidt, M. (1967) Private communication.

\section{DISCUSSION}

L.W. Acton: What do you mean by 'evolution model'?

R.J. Weymann: As we observe objects farther away we observe some systems at an earlier stage of evolution and presumably at earlier stages they are stronger X-ray emitters.

Anonymous: What was the value you used for the intensity of the diffuse cosmic X-ray flux?

R.J. Weymann: I used a value of $10^{-7} \mathrm{ergs} / \mathrm{cm}^{2} \mathrm{sec}^{-1}$ between 1 and 10 angstroms. I believe that is the value that Dr. Friedman has quoted you. 
Ja.B. Zel'dovič: What areal resolution is required to tell the difference between the continuous and discrete models?

R.J. Weymann: The point of the statistical analysis was really to find out how small does the detector scan beam have to be in order to be able to see if these really are discrete sources. It is my guess that even 1 square degree will not be sufficiently small. 